\title{
Factorization of a Class of Perfect Reconstruction Modified DFT Filter Banks with IIR Filters
}

\author{
S. S. Yin and S. C. Chan \\ Department of Electrical and Electronic Engineering \\ The University of Hong Kong, Pokfulam Road, Hong Kong
}

\begin{abstract}
This paper proposed a new factorization of a class of perfect reconstruction (PR) causal-stable modified discrete Fourier transform (MDFT) filter bank (FB) with IIR filters, whose prototype filter has identical denominator in their polyphase components. This factorization technique, which is based on the lifting scheme, is also complete for the PR FIR MDFT FB. It can be applied to convert a nearly PR MDFT FBs to a structural PR system, which is very useful to their multiplier-less realization because the PR property in these structural FBs is unaffected by coefficient quantization. Therefore, it is possible to employ canonical signed digits (CSD) or sum of powers of two coefficients to approximate the coefficients in the factored form without changing the PR property.
\end{abstract}

\section{Introduction}

Perfect reconstruction filter banks (PR FBs) have important applications in speech, audio, image and array processing. An efficient class of M-channel PR FBs is the discrete Fourier transform (DFT) filter bank, which is wellknown for its computational and design efficiency in realizing channels with equal bandwidth. The condition for which such DFT FBs is perfect reconstruction was reported in $[1,2]$ and the resultant DFT FB is called the modified discrete Fourier transform (MDFT) filter bank. Like the cosine modulated filter banks (CMFBs) $[3,10]$, they offer good frequency characteristics while requiring very low design and implementation complexities. A recent study had shown that MDFT FBs with IIR filters have the potential advantage of realizing PR systems with lower system delay, sharper cutoff and higher stopband attenuation over their FIR counterparts [4]. Like the design of IIR CMFB, the design of IIR MDFT FB is also complicated by the highly nonlinear objective function and PR constraints associated with the IIR analysis filters. In [4], the authors suggested to use the same denominator for the polyphase components of the prototype filter in the MDFT FB so that the PR constraints can be considerably simplified. However, when the number of variables and constraints increases, the optimization procedure is rather sensitive to the initial guess of the prototype filter. In [4], a new method for designing NPR and PR IIR MDFT FBs was also proposed. First of all, a PR FIR MDFT FB with similar specification is designed by nonlinear optimization, which is considerably simpler than designing an MDFT FB with IIR filters. The PR FIR prototype filter is then model reduced to an NPR IIR MDFT
FB by modifying a model reduction technique proposed in [5]. The NPR IIR MDFT FB has a reasonably good reconstruction error and it is employed as the initial guess to constrained nonlinear optimization softwares, such as fmincon from MATLAB, for designing the PR IIR MDFT FB. Design results show that both NPR and PR IIR MDFT FBs with good frequency characteristics and different system delays can be obtained readily by the method [4]. Some advantages of this method are that the stability of the modelreduced filter is guaranteed and the IIR filters so obtained closely approximate the properties of the original FIR filter. In addition, the modified model reduction technique in [6] produces a prototype filter with polyphase components with identical denominator, which considerably simplifies the PR constraints. By using these NPR IIR prototype filters as the initial guesses to the constrained nonlinear optimizer, significantly better convergence speed and reliability over the direct nonlinear optimization is achieved.

In this paper, the factorization of PR IIR MDFT FBs is studied. It can be applied to convert a nearly PR MDFT FBs to a structural PR system, which is very useful to their multiplier-less realization because the PR property in these structural FBs is unaffected by coefficient quantization. Therefore, it is possible to employ canonical signed digits (CSD) or sum of powers of two coefficients to approximate the coefficients in the factored form without changing the PR property. Factorization of the biorthogonal FIR CMFBs has been studied in [7,9,11]. In [7,9], a new factorization for a class of IIR CMFB previously proposed in [8] was developed. This factorization is based on the lifting scheme and it is also complete for PR FIR CMFBs and two-channel PR IIR filter banks if the determinant of the polyphase matrix is equal to a constant multiple of signal delays. Since the unconstrained optimization using the lifting coefficients results in highly nonlinear objective function, the design problem is formulated as a constrained optimization in the filter coefficients. The factorization technique is then applied to convert it to a structural PR system. Like the lifting scheme, it also can reduce the arithmetic complexity of the filter bank asymptotically by a factor of two.

The paper is organized as follows: Section II is devoted to the theory of PR FIR MDFT FBs. The theory and design of PR IIR MDFT FBs is recalled in Section III and the proposed factorization is described in Section IV following 
by an example in Section V. Conclusions are drawn in Section VI.

\section{PR FIR MDFT FBS}

The theory of MDFT FBs will be recalled briefly here. Interested readers are referred to [1,2] for more detail. Assume all analysis and synthesis filters in this paper are derived from an identical real-valued low-pass prototype filter $h(n)$ which has a transition band from $-\pi / M$ to $\pi / M$. The complex analysis and synthesis filters for type-I and type-II MDFT FBs are:

$$
h_{k}(n)=f_{k}(n)=\sqrt{2} h(n) \exp \left(j \frac{\pi k}{M}\left(n-\frac{D}{2}\right)\right)
$$

and

$$
\begin{aligned}
& h_{k}(n)=\sqrt{2} h(n) \exp \left(j \frac{\pi k}{M}\left(n-\frac{D+M}{2}\right)\right) \\
& f_{k}(n)=\sqrt{2} h(n) \exp \left(j \frac{\pi k}{M}\left(n-\frac{D-M}{2}\right)\right)
\end{aligned}
$$

respectively, where $k=0, \ldots, 2 M-1$ and $n=0, \ldots, N-1$. It is clear that the two types of MDFT FBs differ in the phase of the modulation and the way the real and imaginary parts are taken to form the subbands. For simplicity, we consider an $2 M$-channel MDFT FB with an overall delay of $D=(2 s+1) M-1$. The PR condition on the polyphase components of the prototype filters for the two types of MDFT FBs are the same:

$$
G_{k}(z) G_{2 M-k-1}(z)+G_{M+k}(z) G_{M-k-1}(z)=c z^{-s}
$$

where $0 \leq k \leq M / 2-1, c$ is a constant, $s$ is an integer and $G_{k}(z)$ are the type-I order-2M polyphase components of the prototype filter $h(n)$. It is equivalent to the PR condition for an $M$-channel biorthogonal CMFB with a system delay of $D_{c m f b}=2 s M-1$. If $s=N /(2 M)$, the prototype filter $h(n)$ is linear phase.

\section{PR IIR MDFT FBS}

Assume that the polyphase components of the IIR prototype filter takes on the following form:

$$
G_{k}(n)=\frac{N_{k}(z)}{D(z)}, k=0, \ldots, M / 2-1,
$$

that is, they have an identical denominator. Hence, the PR condition is (3) will reduce to

$$
N_{k}(z) N_{2 M-k-1}(z)+N_{M+k}(z) N_{M-k-1}(z)=c z^{-s} D^{2}(z),
$$

where $k=0, \ldots, M / 2-1$. It is equivalent to the PR condition for the IIR CMFBs studied in [8]. Also, to ensure that analysis and synthesis filters are stable, all the roots of $D(z)$ shall remain inside the unit circle.
Similar to CMFBs, MDFT FBs are obtained by frequency shifting of the prototype filter. To achieve a good frequency characteristic, the stopband error of the prototype filter needs to be minimized. This leads to the following objective function:

$$
\Phi=\int_{\omega_{s}}^{\pi}\left|H\left(e^{j w}\right)\right|^{d} d \omega
$$

where $\omega_{s}$ is the stopband cutoff frequency of the prototype filter. For $d=2$, the objective function is the familiar least squares design criterion. If approximate equip-ripple passband and stopband errors are desired, the value of $d$ can be chosen as 4 .

Let the z-transform of the IIR prototype filter be:

$$
H(z)=\frac{\sum_{n=0}^{N_{n}-1} a(n) z^{-n}}{\sum_{n=0}^{N_{d}-1} b(n) z^{-n}}, k=0, \ldots, M / 2-1,
$$

where $N_{n}$ and $N_{d}$ are respectively the lengths of the numerator and the denominator of the prototype filter. It is easy to show that if the type-I polyphase components of the prototype filter have the identical denominator, then $b(n)=0$ whenever $n \neq 2 k M+1$, and

$$
H(z)=\sum_{k=0}^{M-1} z^{-k} \frac{N_{k}(z)}{D(z)}=\frac{\sum_{k=0}^{M-1} z^{-k} N_{k}(z)}{D(z)} .
$$

The PR condition in (5) suggests that the length of denominator in the polyphase components should not be longer than the length of the numerators, otherwise it would be very difficult to balance the various powers of $\mathrm{z}$ on both sides of (5). The design problem can be formulated as a constrained optimization problem where (6) is minimized subject to the PR and stability constraints in (5).

Although the use of identical denominator greatly simplifies the design procedure, the optimization procedure is still sensitive to the initial guess of the prototype filter when the number of variables and constraints increases. To overcome this problem, a PR FIR MDFT FB with similar specification is first designed. The PR FIR prototype filter is then model reduced to an NPR IIR MDFT FB by modifying a model reduction technique proposed in [5]. The resulting NPR IIR MDFT FB will have a similar frequency characteristic as the PR FIR counterpart and reasonably good reconstruction error. It can therefore be employed as the initial guess to constrained nonlinear optimization softwares, such as fmincon form MATLAB, for designing the PR IIR MDFT FB. The IIR FB so obtained has a better performance than its FIR filter, especially when the transition bandwidth and system delay are reduced. Normally, it is not difficult to obtain a PR violation as low as $10^{-15}$ for the final IIR MDFT FB. It can therefore be factored to a structural PR system, to be discussed below. 


\section{FACTORIZATION OF M-CHANNEL PR MDFT FBS}

Following the factorization of IIR CMFBs in [7,11], the PR condition of PR IIR MDFT FBs can be viewed as an ideal $\left.\left\{\phi_{0}: \phi_{0}(z)=A(z) N_{k}(z)+B(z) N_{M+k}(z)\right\}=c z^{-s} D^{2}(z)\right\}$ generated by $N_{k}(z)$ and $N_{M+k}(z)$. For simplicity, assume that the common factors of $N_{k}(z)$ and $N_{M+k}(z)$, which must divide the right hand side of (5), have already been removed and they are co-prime to each other. The general solutions of $N_{M-k-1}(z)$ and $N_{2 M-k-1}(z)$ in (5) are given by

$$
\begin{gathered}
N_{M-k-1}(z)=\tilde{N}_{M-k-1}(z)-Q_{k}(z) N_{k}(z) \\
N_{2 M-k-1}(z)=\tilde{N}_{2 M-k-1}(z)+Q_{k}(z) N_{M+k}(z)
\end{gathered}
$$

for some $\tilde{N}_{M-k-1}(z)$ and $\tilde{N}_{2 M-k-1}(z)$ in the ideal $\phi_{0}(z)$, and some $Q(z)$ in the field $F[z]$. Dividing both sides of (9) by $D(z)$, we have

$$
\begin{gathered}
\widetilde{G}_{M-k-1}(z)=\widetilde{G}_{M-k-1}(z)-Q_{k}(z) G_{k}(z) \\
\widetilde{G}_{2 M-k-1}(z)=\widetilde{G}_{2 M-k-1}(z)+Q_{k}(z) G_{M+k}(z)
\end{gathered}
$$

and

$$
\begin{aligned}
& {\left[\begin{array}{cc}
G_{k}(z) & G_{M-k-1}(z) \\
-G_{M+k}(z) & G_{2 M-k-1}(z)
\end{array}\right]} \\
& \quad=\left[\begin{array}{cc}
G_{k}(z) & \widetilde{G}_{M-k-1}(z) \\
-G_{M+k}(z) & \widetilde{G}_{2 M-k-1}(z)
\end{array}\right]\left[\begin{array}{cc}
1 & -Q_{k}(z) \\
0 & 1
\end{array}\right]
\end{aligned}
$$

$\widetilde{G}_{M-k-1}(z)$ and $\widetilde{G}_{2 M-k-1}(z)$ are particular solution to (3). By using the Euclidean algorithm, $N_{k}(z)$ and $N_{M+k}(z)$, since they are co-prime, can be written as

$$
\left[\begin{array}{c}
N_{k}(z) \\
-N_{M+k}(z)
\end{array}\right]=\prod_{i=1}^{n_{l}}\left[\begin{array}{cc}
q_{i}(z) & z^{-d_{i}} \\
1 & 0
\end{array}\right]\left[\begin{array}{c}
K z^{-d_{0}} \\
0
\end{array}\right]
$$

where $K$ is a non-zero constant. Dividing both sides of (12) by $D(z)$, we have

$$
\left[\begin{array}{c}
G_{k}(z) \\
-G_{M+k}(z)
\end{array}\right]=\prod_{i=1}^{n_{l}}\left[\begin{array}{cc}
q_{i}(z) & z^{-d_{i}} \\
1 & 0
\end{array}\right]\left[\begin{array}{c}
K_{l} z^{-d_{0}} / D(z) \\
0
\end{array}\right] .
$$

Then, a particular solution for $\widetilde{G}_{M-k-1}(z)$ and $\widetilde{G}_{2 M-k-1}(z)$ can be constructed as follows

$$
\begin{aligned}
& {\left[\begin{array}{cc}
G_{k}(z) & \widetilde{G}_{M-k-1}(z) \\
-G_{M+k}(z) & \widetilde{G}_{2 M-k-1}(z)
\end{array}\right]} \\
& \quad=\prod_{i=1}^{n_{l}}\left[\begin{array}{cc}
q_{i}(z) & z^{-d_{i}} \\
1 & 0
\end{array}\right]\left[\begin{array}{cc}
K z^{-d_{0}} / D(z) & 0 \\
0 & (-1)^{n_{l}} c z^{-\left(s-d_{c}\right)} D(z) / K
\end{array}\right]
\end{aligned}
$$

where $c$ and $d_{c}$ are respectively the scale factor and the sum of the delays generated during the division of the Laurent polynomials. So the desired solution is

$$
\begin{aligned}
& {\left[\begin{array}{cc}
G_{k}(z) & G_{M-k-1}(z) \\
-G_{M+k}(z) & G_{2 M-k-1}(z)
\end{array}\right]} \\
& \quad=\prod_{i=1}^{n_{l}}\left[\begin{array}{cc}
q_{i}(z) & z^{-d_{i}} \\
1 & 0
\end{array}\right]\left[\begin{array}{cc}
K z^{-d_{0}} / D(z) & -\widetilde{Q}_{k}(z) / D(z) \\
0 & (-1)^{n_{l}} c z^{-\left(s-d_{c}\right)} D(z) / K
\end{array}\right]
\end{aligned}
$$

where $\widetilde{Q}_{k}(z)=K Q_{k}(z)$. It should be noted that in the FIR case, $D(z)=1$. Therefore, this factorization is also applicable to the FIR MDFT FBs and type II MDFT FBs. Note that the factorization in (12) is non-unique and it is possible to eliminate either the highest or lowest powers of the Laurent polynomial. In this paper, this option is employed to obtain a factorization with a lower dynamic range in its coefficients.

\section{EXAMPLE}

In this section, we will give an example to show the efficiency of the factorization proposed. A 4-channel PR FIR biorthogonal MDFT FB with a length $N=m M=5 \times 4=20$ was first designed by nonlinear optimization. The total system delay of the 4-channel FIR MDFT FB

\section{is} $D_{m d f t_{-} f b}=(2 s+1) M / 2-1=(2 \times 3+1) \times 2-1=13 \quad$ samples. After the PR FIR MDFT FB was designed, the modified model reduction $[4,6]$ was then used to model reduce the FIR MDFT FB to an NPR IIR MDFT FB with similar frequency characteristics. Using the prototype filter of the NPR IIR MDFT FB so obtained as the initial guess, a PR IIR MDFT FB with the similar specification can be obtained by solving a constrained nonlinear optimization problem. The coefficients of the numerator and the denominator of the IIR prototype filter obtained are listed in table I. The factorization proposed in section IV was then applied to this PR IIR MDFT FB and the coefficients of the factorization are given in Table 2 .

To avoid large dynamic range of the coefficients in the factorization, $q_{3}(z)$ is obtained by removing both the lowest and the highest power of $z^{-1}$, that is, $z^{0}$ and $z^{-2}$. This gives $d_{c}=1$ in the matrix factor of $q_{3}(z)$. This is obtained by searching the possible factorizations for coefficients with low dynamic range.

$$
\begin{aligned}
& {\left[\begin{array}{cc}
N_{1}(z) & N_{2}(z) \\
-N_{3}(z) & N_{4}(z)
\end{array}\right]} \\
& =\prod_{i=1}^{2}\left[\begin{array}{cc}
q_{i}(z) & 1 \\
1 & 0
\end{array}\right]\left[\begin{array}{cc}
q_{3}(z) & z^{-1} \\
1 & 0
\end{array}\right]\left[\begin{array}{cc}
q_{4}(z) & 1 \\
1 & 0
\end{array}\right]\left[\begin{array}{cc}
K & -Q^{\prime}(z) \\
0 & c z^{-1} / K
\end{array}\right] .
\end{aligned}
$$

The frequency responses of the analysis filter banks before and after factorization are shown in Fig. 1(a) and 1(b), respectively. It can be seen that the frequency response of the PR system is very close to the nearly PR system after optimization. The latter has a PR violation of the order 
$10^{-15}$. The smaller the PR violation, the closer will the factored FB to its nearly PR version.

TABLE I. COEFFICIENTS OF THE IIR PROTOTYPE FILTER

\begin{tabular}{|c|c|l|c|}
\hline \multicolumn{4}{|c|}{ Coefficients of numerator } \\
\hline \multirow{4}{*}{$N_{1}(z)$} & -0.00373633968267 & & -0.00947433498353 \\
& 0.17432052918835 & $N_{3}(z)$ & 0.29599743806411 \\
& 0.09176840168736 & & -0.05521523117019 \\
& -0.00985074712100 & & 0.00283859339749 \\
\hline \multirow{4}{*}{$N_{2}(z)$} & -0.02288183380604 & & 0.05981538177822 \\
& 0.27043453622126 & \multirow{2}{*}{$N_{4}(z)$} & 0.22251382040226 \\
& -0.01313803466332 & & -0.03967320266941 \\
& 0.01264518725457 & & 0.00385979165614 \\
\hline
\end{tabular}

\begin{tabular}{|l|c|}
\hline \multicolumn{2}{|c|}{ Coefficients of denominator } \\
\hline \multirow{3}{*}{$D(z)$} & 1.00000000000000 \\
& -0.01834999387356 \\
& 0.0000000000000 \\
0.00000000020258 \\
\hline
\end{tabular}

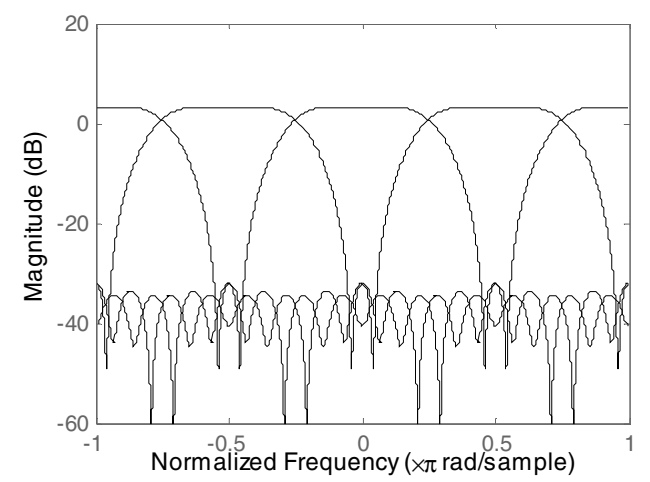

(a)

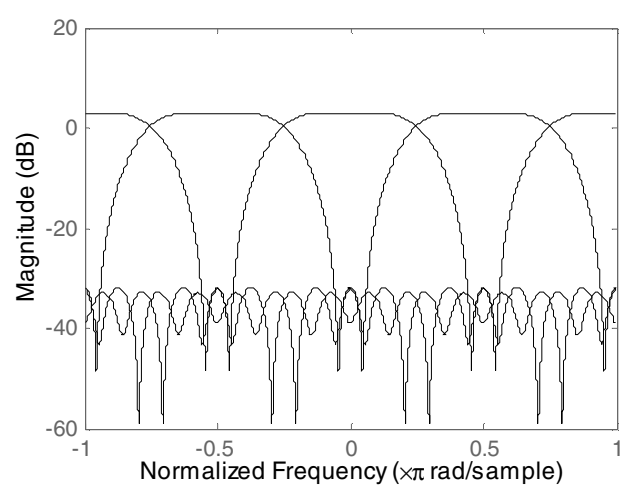

(b)

Figure 1. The frequency responses of (a) analysis filter bank before factorization and (b) analysis filter bank after factorization
TABLE II. COEFFICIENTS OF THE FACTORIZATION

\begin{tabular}{|c|c|c|}
\hline & $z^{0}$ & $z^{-1}$ \\
\hline$q_{1}(z)$ & 3.27612703851365 & 0 \\
\hline$q_{2}(z)$ & -0.20457826897225 & 0.03303591540458 \\
\hline$q_{3}(z)$ & -14.54646033651212 & 1.39077673176299 \\
\hline$q_{4}(z)$ & 0.01004380235816 & -0.26040131543809 \\
\hline$K$ & 0.23725548037474 & 0 \\
\hline$-Q^{\prime}(z)$ & 1.49789163696030 & -0.08127204296356 \\
\hline$c$ & 0.13154856295746 & 0 \\
\hline
\end{tabular}

\section{CONCLUSIONS}

A new factorization of a class of PR causal-stable MDFT FB with IIR filters is presentation. This factorization technique, which is based on the lifting scheme, is also complete for the PR FIR MDFT FB. It can be applied to convert a nearly PR MDFT FBs to a structural PR system, which is very useful to their multiplier-less realization because the PR property in these structural FBs is unaffected by coefficient quantization. A design example is given to illustrate the effectiveness of the approach.

\section{References}

[1] P. N. Heller, T. Karp and T. Q. Nguyen, "A gemera; formulation of modulated filter banks," IEEE Trans. on SP, vol. 47, No. 4, pp.9861002, April 1999.

[2] T. Karp and N. J. Fliege, "Modified DFT filter banks with perfect reconstruction," IEEE Trans. on Circuits and systems, vol. 46, No. 11. pp.1404-1414, Nov. 1999.

[3] P. P. Vaidynathan, Multirate systems and filter banks, Englewood cliffs, NJ: Prentice Hall, 1992.

[4] S. S. Yin and S. C. Chan, "The theory and design of a class of perfect reconstruction modified DFT filter banks with IIR filters," in Proc. IEEE 2004 Midwest Symposium on Circuits and Systems, vol. III, pp. 203-206, July 25-28, 2004.

[5] H. Brandenstein and R. Unbehauen, "Least-squares approximation of FIR by IIR filters," IEEE Trans. on SP, vol. 46, no. 1, pp.21-30, Jan. 1998.

[6] S. C. Chan, K. M. Tsui and K. W. Tse, "Design of constrained IIR and interpolated IIR filters using a new semi-definite programming based model reduction technique," in Proc. Eusipco2004.

[7] S. C. Chan, J. S. Mao, P. M. Yiu and K. L. Ho, "The factorization of M-channel FIR and IIR cosine-modulated filter banks and their multiplier-less realization using SOPOT coefficients," Proc. IEEE 2004 Midwest Symposium on Circuits and Systems, vol. II, pp. 109112, July 25-28, 2004.

[8] J. S. Mao, S. C. Chan and K. L. Ho, "Theory and design of a class of M-channel IIR cosine-modulater filter banks," IEEE Signal Processing Letters, Vol. 7, No. 2, pp.38-40, Feb. 2000.

[9] T. Karp, A. Mertins and G. Schuller, "Efficient biorthogonal cosinemodulated filter banks," EURASP Signal Processing, vo; 81, pp. 9971016, May 2001.

[10] R. D. Koilpillai and P. P. Vaidyanathan, "Cosine-modulated FIR filter banks satisfying perfect reconstruction," IEEE Trans. on SP, vol. 40, pp. 770-783, Apr. 1992.

[11] J. S. Mao, "New design and factorization methods for perfect reconstruction filter banks," Ph.D thesis, Dept. of Elect \& Electronic Engg., The University of Hong Kong, Apr. 2000 